\title{
Common 22-year cycles of Earth rotation and solar activity
}

\author{
Yavor Chapanov ${ }^{1}$, Jan Vondrák ${ }^{2}$ and Cyril Ron ${ }^{2}$ \\ ${ }^{1}$ Central Laboratory for Geodesy, Bulgarian Academy of Sciences \\ Akad. G. Bonchev Str. Bl.1, Sofia 1113, Bulgaria \\ email:chapanov@clg.bas.bg \\ ${ }^{2}$ Astronomical Institute, Academy of Sciences of Czech Republic \\ Boční II, 14131 Prague, Czech Republic \\ email: vondrak@ig.cas.cz, ron@ig.cas.cz
}

\begin{abstract}
The 22-year oscillations of the Earth rotation due to several geophysical processes in the core-mantle system, oceans, atmosphere and geomagnetic field are excited mainly by 22year cycles of the solar activity. These geophysical processes produce their own oscillations of the Earth rotation with different periods around 22 years. The direct and indirect influence of the solar activity on 22-year cycles of the Earth rotation are separated from the core effects and corresponding amplitudes are estimated by means of two approaches. The first, direct approach uses extended time series of Wolf's numbers with 22-year cycles, determined by sign alternation of even sunspot cycles. A linear regression between 22-year cycles of UT1 and solar activity is determined and this regression model is used to calculate the UT1 response to the 22-year cycles of the solar activity. The second, indirect approach uses 22-year oscillation of the mean sea level, caused by water evaporation due to variations of the total solar irradiance. The influence of the mean sea level variations on the Earth rotation is calculated by means of an empirical model of global water redistribution. The core-mantle effects on the 22-year UT1 variations are determined by excluding the UT1 response to the solar activity and core angular momentum due to the geomagnetic field variations, according to the solutions from the Special Bureau of the Core (SBC).
\end{abstract}

Keywords. Sun: activity, sunspots, solar-terrestrial relations, time

\section{Introduction}

The variations of the Earth rotation are mainly caused by the influence of the Moon, Sun and planets, displacements of matter in different parts of the planet and other excitation mechanisms. The most powerful source of these oscillations is Sun, which solar activity cycles can drive great number of geodynamical processes connected with the convections of the Earth fluids on the surface and inside the Earth. Many of climate and weather parameters are affected directly by the variations of the solar activity. The common 22-year cycles of Earth rotation, solar activity and mean sea level are investigated here by means of the existing long time series of the Universal Time UT1 (1623-2005), based on the solution of Stephenson \& Morrison (1984); the Wolf's numbers since 1749 and the Mean Sea Level (MSL) in Stockholm since 1774 (Ekman 2003).

\section{Common 22-year cycles of UT1 and mean sea level}

A significant part of the UT1 oscillations with period 22 years are excited by the climatic variations, due to the solar activity cycles with the same period. The energy 
source of this process is the Total Solar Irradiance (TSI) variations with maximal amplitude $0.2 \mathrm{~W} / \mathrm{m}^{2}$, which provide an additional water evaporation and global mean sea level oscillations, synchronized with the global water redistribution and corresponding change of the ice thickness at the polar caps (Chapanov \& Gambis 2008). The common cycles of the UT1 and Mean Sea Level (MSL) variations are extracted from the Fourier approximation of the available data for the period 1800-2000 and combination of the oscillations with periods from band 20-25a (Fig. 1,a, Chapanov et al. 2008). UT1 data from star occultation and MSL data from Stockholm maregraph are used. The 22-year MSL and the delayed UT1 cycles are highly correlated with coefficient -0.81 and linear regression (Fig. 1,b) $\Delta \mathrm{UT} 1=-41 \Delta \mathrm{MSL}+2.4[\mathrm{~ms} / \mathrm{mm}]$. This result points out to some dependence between the MSL variations and total UT1 oscillations, dominated mainly by the liquid Earth core effects. It is possible to determine UT1 response to 22-year solar cycles by applying a regression coefficient $-17.5 \mathrm{~ms} / \mathrm{mm}$ to the MSL variations. This coefficient is determined in (Chapanov \& Gambis 2009) with accounting the proportion of $70 \%$ of evaporated water, redistributed over the polar ice and $30 \%$ - over the continents. The resulting time series is shown in Fig. 1,c together with the 22-year core effects on UT1 oscillations, determined from the total UT1 variations after excluding the solar activity influences.

(a) 18001850190019502000

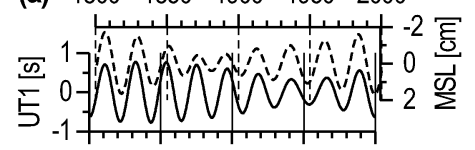

Epoch 18001850190019502000 (b) $\quad-20-100 \quad 1020$

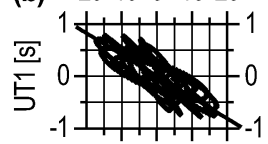

MSL [mm] $-10010 \quad 1020$ (c) $1800185019001950 \quad 2000$

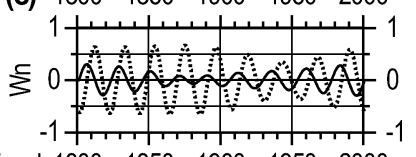

Epoch 18001850190019502000

Figure 1. Common 22-year UT1 (solid line) and MSL (dashed line) cycles for the period 1800-2000 - (a); their linear regression - (b); separation of 22-year cycles of the Earth rotation, due to TSI variations (solid line) and liquid Earth core effects (dotted line) - (c).

\section{Common 22-year cycles of UT1 and extended Wolf's numbers}

The separation of 22-year cycles of the Earth rotation, due to solar activity and core effects by means of MSL data, shown in Fig. 1,c yields rather smoothed time series with no high-frequency details. It is possible to improve the resolution by means of extended 22-year Wolf's numbers cycles $W_{n / 22 a}$ ) determined by sign alternation of 11-year Wolf's numbers cycles (Fig. 2,a).
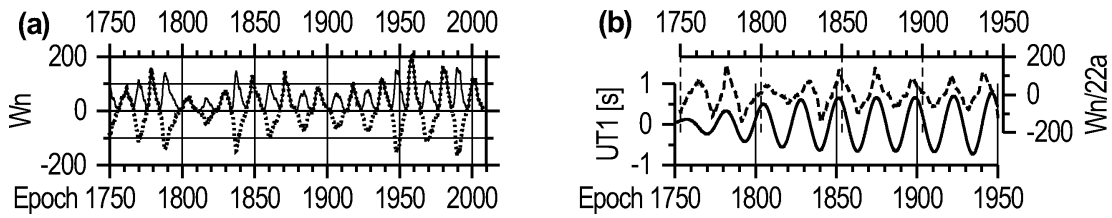

Figure 2. Extended time series of 22-year solar activity cycles (dotted line), determined by sign alternation of Wolf's numbers cycles (solid line) - (a); comparison between the 22-year cycles of UT1 (solid line) and extended Wolf's numbers (dashed line) for the periods 1750-2005 - (b).

Comparison between the 22-year cycles of UT1 and extended Wolf's numbers shows good agreement for the periods 1750-2005 (Fig. 2,b), where the linear regression is $\Delta \mathrm{UT} 1=6.4 W_{n / 22 a}+1.1[\mathrm{~ms}]$ (Fig. 3,c). This regression reflects the possible dependence between the solar activity and total UT1 oscillations, dominated mainly by the liquid Earth core effects. The UT1 response to 22-year solar cycles is determined by linear regression between the MSL and Wn/22a variations for the period 1800-2000 (Fig. 5) 
$\Delta \mathrm{MSL}=-0.074 W_{n / 22 a}+0.26[\mathrm{~mm}]$, and according to the results in (Chapanov \& Gambis 2009 ), the final regression coefficient between UT1 variations and $W_{n / 22 a}$ is $1.3 \mathrm{~ms}$. The separated solar and core effects of 22-year UT1 oscillations are presented in Fig. 3,d.

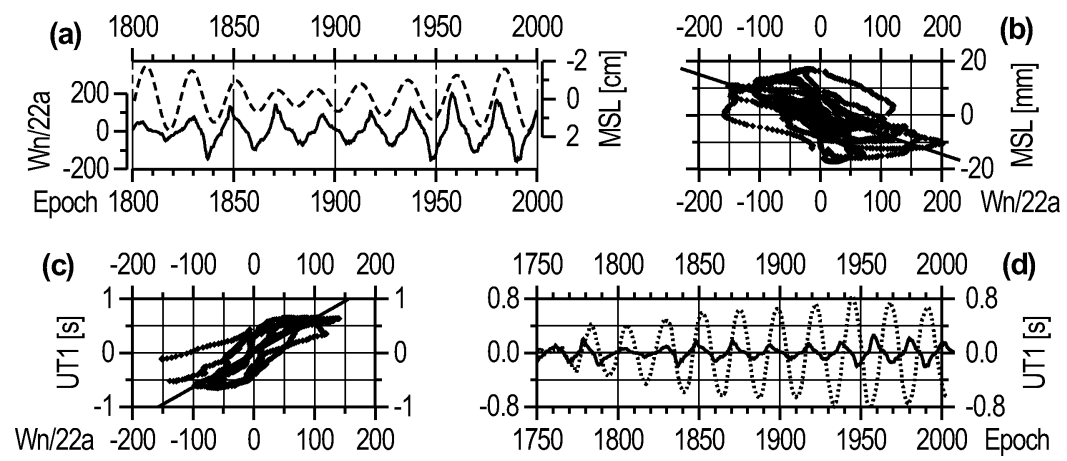

Figure 3. Comparison between $W_{n / 22 a}$ (solid line) and 22-year cycles of MSL (dashed line) (a); their linear regression - (b); linear regression between UT1 and $W_{n / 22 a}$ - (c); separation of 22-year cycles of UT1, due to solar activity (solid line) and liquid Earth core effects (dotted line) - (d).

\section{Conclusions}

- The influence of 22-year cycles of the solar activity on the Earth rotation variations is determine by analysis of two time series - first with the mean sea level data from Stockholm maregraph station for the period 1800-2000 and second - with an artificial Index of 22-year solar cycles, based on the Wolf's numbers for the period 1750-2005. The mean sea level data analysis is more realistic, because the long-term MSL oscillations are physically connected with additional water evaporation due to the total solar irradiance variations and global water redistribution over the continents and polar ice, following by the changes of the Earth axial moment of inertia and Earth rotation velocity. The extended 22-year Wolf's numbers Index provide longer data, more short-term details and exact match of cycles duration, but it need future improvement in order to represent more realistic the amplitude of the TSI variations.

- The direct influence of 22-year solar cycles on the Earth rotation variations, determined by means of the mean sea level data for the period 1800-2000, is with slow variable amplitude of UT1 cycles and maximal value of about $0.3 \mathrm{~s}$. The core effects in 22-year UT1 oscillations are with constant amplitude of about $0.66 \mathrm{~s}$ for the period $1800-1890$ and non-linear changes after 1890; close frequency to the solar cycles and constant phase difference of about 65 degrees.

\section{Acknowledgements}

The work was supported by grants No.DO 02-275 with Bulgarian NSF and No.A300130702 with Grant Agency of Acad. of Sci. of the Czech Republic.

\section{References}

Chapanov, Ya. \& Gambis, D. 2008, Journées 2008 "Syst. de ref. spat.-temp.", Dresden, 131-132 Chapanov, Ya. \& Gambis, D. 2009, Proc. IAU Symposium 264, This Issue

Chapanov, Ya., Vondrák, J., \& Ron, C. 2008, Journées 2008 "Syst. de ref. spat.-temp.", Dresden, $178-179$

Ekman, M. 2003, Small Publ. in Hist. Geophys., 12, 31

Stephenson, F. R. \& Morrison, L. V. 1984, Phil. Trans. R. Soc. of London, Ser. A., 313, 47-70 